УДК 532.6

DOI $10.18101 / 2306-2363-2020-2-22-27$

\title{
ИССЛЕДОВАНИЕ СНАРЯДНОГО РЕЖИМА ТЕЧЕНИЯ ВОДЫ И НЕФТИ В Ү-МИКРОКАНАЛЕ
}

() Пряжников А. И.

младший научный сотрудник,

Сибирский федеральный университет

660041, Красноярск, пр. Свободный, 79

apryazhnikov@sfu-kras.ru

\section{(C) Пряжников М. И.}

научный сотрудник,

Сибирский федеральный университет

660041, Красноярск, пр. Свободный, 79

E-mail: mpryazhnikov@sfu-kras.ru

(C) Якимов А. С.

младший научный сотрудник,

Сибирский федеральный университет

660041, Красноярск, пр. Свободный, 79

asyakimov@gmail.com

\section{(С) Гузей Д. В.}

научный сотрудник,

Сибирский федеральный университет

660041, Красноярск, пр. Свободный, 79

E-mail: dguzei@sfu-kras.ru

(C) Дамдинов Б. Б.

доктор физико-математических наук, профессор,

Сибирский федеральный университет

660041, Красноярск, пр. Свободный, 79

Бурятский государственный университет имени Доржи Банзарова

670000, Улан-Удэ, ул. Смолина, 24а

bdamdinov@sfu-kras.ru

(C) Жигарев В. А.

научный сотрудник,

Сибирский федеральный университет

660041, Красноярск, пр. Свободный, 79

vzhigarev@sfu-kras.ru

\section{Для цитирования:}

Пряжников А. И., Пряжников М. И., Якимов А. С., Гузей Д. В., Дамдинов Б. Б., Жигарев B. А. Исследование снарядного режима течения воды и нефти в Ү-микроканале // Вестник Бурятского государственного университета. Химия. Физика. 2020. Вып. 2. C. 22-27. 
А. И. Пряжников, М. И. Пряжников, А. С. Якимов, Д. В. Гузей, Б. Б. Дамдинов, В. А. Жигарев. Исследование снарядного режима течения воды и нефти в Ү-микроканале

Работа посвящена изучению снарядного режима течения несмешивающихся жидкостей вода-нефть. Проведено экспериментальное изучение снарядного режима течения воды и нефти в Y-микроканале в широком диапазоне расходов. Проведено численное моделирование, а также сопоставление результатов расчёта с экспериментом. Показано, что моделирование качественно верно описывает форму и размер снарядов, а также расстояние между ними. Определены диапазоны, в которых возможен снарядный режим течения вода-нефть. Установлена зависимость длины снарядов воды от отношения расходов жидкостей. Показано экспериментально, что расчет во всех рассмотренных режимах качественно предсказывает форму границы раздела между жидкостями.

Ключевые слова: несмешивающиеся жидкости; границы раздела фаз; Ү-микроканал; снарядный режим течения; численное моделирование; модель VOF; смачиваемость.

\section{Введение}

Повышение нефтеотдачи является весьма актуальной задачей. Улучшение нефтеотдачи в терригенных породах (сложенных, например, песчаником) во многом определяется структурой течения в поровом пространстве. Режимы течения, возникающие в микропористой среде, зависит от нескольких параметров, а именно от геометрии (характерный размер и тип соединений микроканалов пористой среды), физических свойств нефти и вытесняющей жидкости (плотность, вязкость, межфазное натяжения, краевой угол смачивания). Течения двухкомпонентных несмешивающихся жидкостей (система вода-нефть является таковой) широко распространены [1]. Для практических приложений важно знать диапазон расходов, в котором реализуется тот или иной режим течения, а также его гидродинамические характеристики.

В данной работе проведено экспериментальное исследование и численное моделирование снарядного режима течения двухкомпонентной системы вода-нефть в Y-микроканале. Проведено сравнение результатов численного моделирования с экспериментальными данными по картинам течения.

\section{Экспериментальное исследование}

В работе использовалась дистиллированная вода и нефть. Плотность нефти

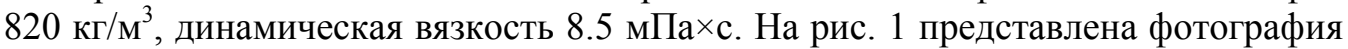
чипа и Y-микроканала под микроскопом. Чип был изготовлен методом фрезерования из ПММА [2]. Ширина и высота микроканала составляла 500 и 200 мкм соответственно.
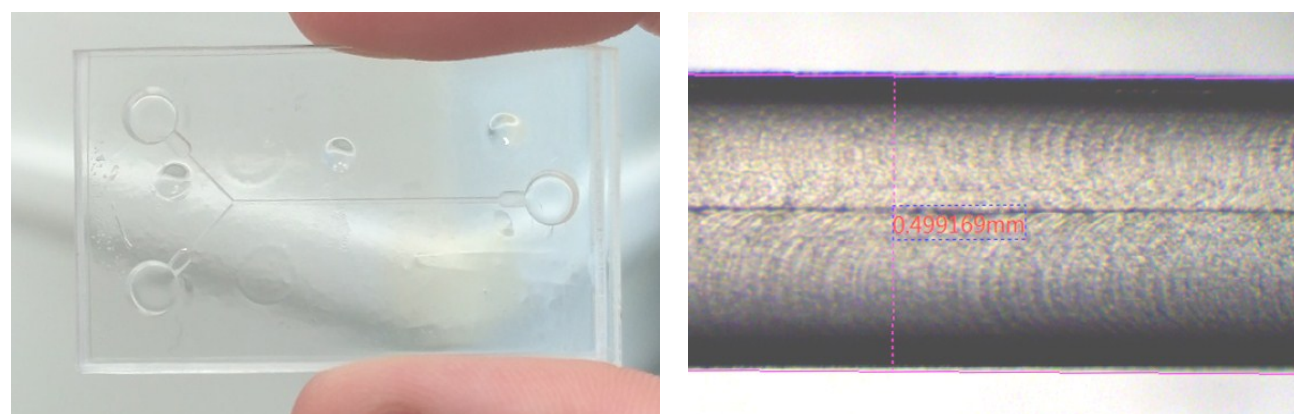

Рис. 1. Фотография чипа (сверху) и микроканала под микроскопом (снизу) 
Входы микроканала соединялись трубкой (с внутренним диаметром 1 мм) со шприцами (Гамильтон, 5мл). Подача жидкостей осуществлялась шприцевым насосом SP lab02. Картина течений фиксировалась камерой ТоupCam и микроскопом Микромед.

\section{Численное моделирование}

Для моделирования течения системы вода-нефть в Y-микроканале использовалась численная методика, основанная на методе VOF (Volume of Fluid - VOF) [3] и представленная в работе [4]. Суть метода VOF заключается в рассмотрении системы вода-нефть как единой двухкомпонентной среды. Пространственное распределение фаз в пределах расчетной области определяется при помощи специальной функции маркера $F(x, y, z, t)$, величина которой задает объемную долю жидкой фазы в расчетной ячейке следующим образом: $F(x, y, z, t)=0$ если ячейка пустая, $F(x, y, z, t)=1$ если ячейка полностью заполнена жидкостью.

Поскольку свободная поверхность движется вместе с жидкостью, то отслеживание перемещения свободной границы в пространстве осуществляется путем решения уравнения переноса объемной доли жидкой фазы в ячейке:

$$
\frac{d F}{d t}+V \cdot \nabla F=0
$$

где $V$ - вектор скорости двухфазной среды, найденный из решения системы уравнений гидродинамики, в которую входят уравнение неразрывности (закон сохранения массы), уравнения движения (закон сохранения импульса).

При рассмотрении течений жидкости с границей раздела необходимо учитывать поверхностное натяжение в микроканале. Метод VOF позволяет относительно просто учесть влияние сил поверхностного натяжения. Чаще всего для моделирования поверхностного натяжения в рамках VOF метода используют CSF (Continuum surface force) алгоритм [5], суть которого состоит во введении в уравнения движения дополнительной объемной силы $F_{s}$, величина которой определяется из соотношения:

$$
F_{s}=\sigma k \nabla F
$$

где $\sigma$ - коэффициент поверхностного натяжения, $k-$ кривизна свободной поверхности.

\section{Результаты и обсуждение}

Снарядный режим течения наблюдается при малых расходах воды и нефти. Во всех экспериментах в этом режиме формируются снаряды воды. Нефть является дисперсной (несущей) средой, так как нефть лучше смачивает стенки канала. Вода выступает в качестве дисперсной фазы. Разделение дисперсной фазы на снаряды определяется межфазным натяжением. Снаряд воды занимает почти все поперечное сечение микроканала с очень тонкой плёнкой нефти между снарядом воды и стенкой микроканала. При маленьких расходах обеих жидкостей силы межфазного натяжения начинают доминировать над силами инерции.

При постоянном расходе воды (дисперсной фазы) наблюдалось уменьшение длины снаряда при увеличении расхода нефти (дисперсной среды), как показано 
А. И. Пряжников, М. И. Пряжников, А. С. Якимов, Д. В. Гузей, Б. Б. Дамдинов, В. А. Жигарев. Исследование снарядного режима течения воды и нефти в Ү-микроканале

на рис. 2. Такая же тенденция наблюдалась при постоянном расходе нефти, но при уменьшении расхода воды.

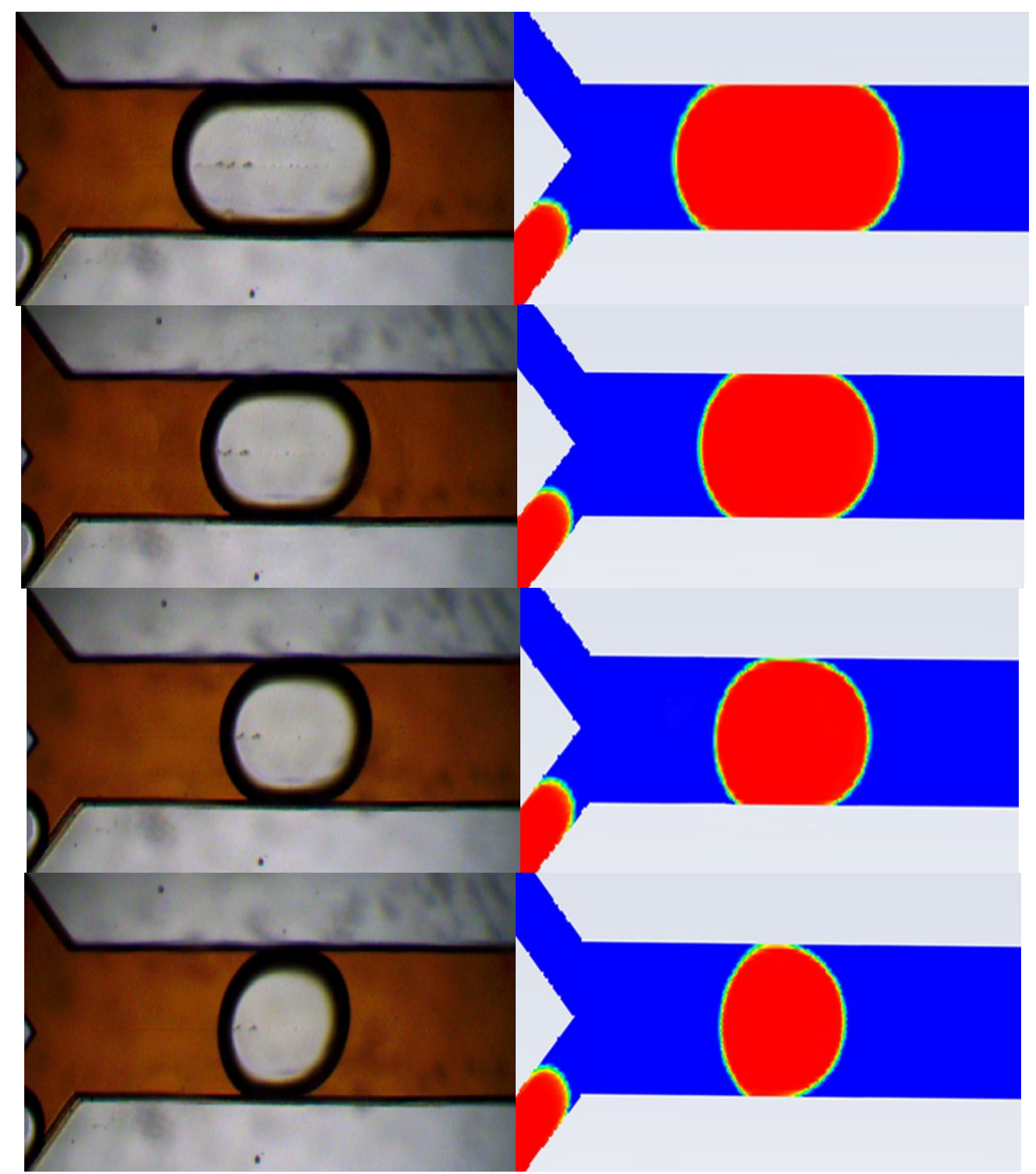

Рис. 2. Снарядный режим при расходе воды 0.067 мл/ч. Расход нефти (сверху вниз), мл/ч: $0.5,3.1,5.5$.

На рис. 2 представлено качественное сравнение результатов эксперимента (слева) и расчета (справа). Как видно, наблюдается хорошее качественное согласие экспериментальных фотографий и результатов моделирования по форме снарядов. 
Установлено, что снарядный режим течения вода-нефть Ү-микроканале реализуется в следующих диапазонах критериев подобия: $\mathrm{Re}_{\mathrm{w}}<10$ и $\mathrm{Re}_{\mathrm{o}}<1.3, \mathrm{We}_{\mathrm{w}}$ $<1.3 \cdot 10^{-2}$ и $\mathrm{We}_{\mathrm{o}}<2.4 \cdot 10^{-2}, \mathrm{Ca}_{\mathrm{w}}<1.3 \cdot 10^{-2}$ и $\mathrm{Ca}_{\mathrm{w}}<1.8 \cdot 10^{-2}$. Отметим, что при очень низких расходах нефти формируются удлинённые снаряды. Под удлинёнными снарядами мы подразумеваем снаряды, длина которых превышает расстояние между ними.

\section{Заключение}

В результате исследования были определены диапазоны, в которых возможен снарядный режим течения вода-нефть. Установлена зависимость длины снарядов воды от отношения расходов жидкостей. Проведенное сравнение расчета и эксперимента показало, что расчет во всех рассмотренных режимах качественно верно предсказывает форму границы раздела между жидкостями, правильно описывает размер и форму снарядов и капель и расстояние между ними.

Работа выполнена в рамках государственного задания Министерства науки и высшего образования РФ (номер FSRZ-2020-0012). При поддержке РФФИ (грант18-02-00523a).

\section{Литература}

1. Kashid M. N., Agar D. W. Hydrodynamics of liquid-liquid slug flow capillary microreactor: flow regimes, slug size and pressure drop // Chem. Eng. J. — 2007. — V. 131. — P. 113.

2. Lukyanenko K. A., Denisov I. A., Sorokin, V. V., Yakimov A. S., Esimbekova E. N., Belobrov P. I. Handheld enzymatic luminescent biosensor for rapid detection of heavy metals in water samples // Chemosensors. — 2019. - V. 7(16). - P. 1-10.

3. Hirt C. W., Nichols B. D. Volume of fluid (VOF) methods for the dynamic of free boundaries // J. Comput. Phys. - 1981. - V. 39. - P. 201-226.

4. Minakov A. V. Numerical algorithm for moving-boundary fluid dynamics problems and its testing // J. Comput. Math. \& Math. Phys. — 2014. - V. 54, № 10. P. - 1560-1570.

5. Brackbill J. U., Kothe D. B., Zemach C. A continuum method for modeling surface tension // J. Comput. Phys. - 1992. - V. 100. - P. 335-354.

\section{INVESTIGATION OF THE SLUG REGIME OF WATER AND OIL FLOW IN THE Y-MICROCHANNEL}

Pryazhnikov A. I.

junior researcher,

Siberian Federal University

660041, Krasnoyarsk, Svobodny pr., 79

apryazhnikov@sfu-kras.ru

Pryazhnikov M. I.

researcher,

Siberian Federal University

660041, Krasnoyarsk, Svobodny pr., 79

mpryazhnikov@sfu-kras.ru 
Yakimov A. S.

junior researcher,

Siberian Federal University

660041, Krasnoyarsk, Svobodny pr., 79

asyakimov@gmail.com

Guzei D. V.

researcher,

Siberian Federal University

660041, Krasnoyarsk, Svobodny pr., 79

dguzei@sfu-kras.ru

Damdinov B. B.

doctor of physics and mathematics, professor

Siberian Federal University

660041, Krasnoyarsk, Svobodny pr., 79

Banzarov Buryat State University

670000, Ulan-Ude, Smolina str., 24a

bdamdinov@sfu-kras.ru

Zhigarev V. A.

researcher,

Siberian Federal University

660041, Krasnoyarsk, Svobodny pr., 79

vzhigarev@sfu-kras.ru

The work is devoted to the study of the slug regime flow of immiscible liquids water-oil. The experimental study of the slug regime of water and oil flow in the Y-microchannel in a wide range of flow rates is carried out. Numerical modeling and comparison of the calculation results with the experiment are carried out. It is shown that modeling qualitatively correctly describes the shape and size of projectiles, as well as the distance between them. The ranges in which the slug regime of the water-oil flow is possible are determined. The dependence of the length of water shells on the ratio of liquid flow rates is established. It is shown experimentally that the calculation in all the considered modes qualitatively predicts the shape of the interface between liquids.

Keywords: immiscible liquids; phase boundaries; Y-microchannel; slug flow regime; numerical simulation; VOF model; wettability.

Статья поступила в редакцию 16.10.2020; принята к публикации 30.10.2020. 